Check for updates

Cite this: RSC Adv., 2019, 9, 26773

\title{
Highly sensitive polyaniline-coated fiber gas sensors for real-time monitoring of ammonia gas $\uparrow$
}

\author{
Naraporn Indarit, ${ }^{a}$ Yong-Hoon Kim, (D) bc Nattasamon Petchsang (D) *de \\ and Rawat Jaisutti ${ }^{\star a}$
}

A single-yarn-based gas sensor has been made from conductive polyaniline coated on commercial yarns. This can detect ammonia gas concentration in an environment or a working area. Cotton, rayon and polyester are utilized as substrates using a dip-coating process. The conductive yarns show ohmic behavior with an electrical resistance of $15-31 \mathrm{k} \Omega \mathrm{cm}^{-1}$. The conductive polyester yarn exhibits higher mechanical strength even after intensive chemical treatment. It also has the highest gas response of $57 \%$ of $50 \mathrm{ppm}$ ammonia gas, the concentration at which health problems will occur. A linear gas response of the yarn sensor appears in a range of 5-25 ppm ammonia concentration. The polyester yarn sensor can be reused without any change in its sensing response. It can monitor gas levels continuously giving real-time results. By using a microcontroller as part of the circuitry, the gas detection results are transferred and updated wirelessly to a computer or to a smartphone. The textile-based gas sensor can be sewn directly onto the fabrics since it is made with the same fabric. This single-yarn-based gas sensor is suitable for mass production and is appropriate for sophisticated applications.

Received 27th May 2019

Accepted 18th August 2019

DOI: $10.1039 / \mathrm{c} 9 \mathrm{ra0} 4005 f$

rsc.li/rsc-advances deal with toxic gas. It would also make them convenient to use onsite.

There have been some investigations on high performance textile-based gas sensors, for example, nanoscale carbon materials $^{7-9}$ and conducting polymers. ${ }^{10-14}$ The conductive polymer is interesting due to its being a highly stretchable conductive material, so it has been used in various applications. ${ }^{\mathbf{1 0}, \mathbf{1 5}-\mathbf{1 8}}$ However, making a polymer-based sensor is complicated because of stringent electrical and mechanical constraints as well as the need to simultaneously detect different oxidizing and reducing gases. Some examples of conductive polymer are polypyrrole,${ }^{14}$ polythiophene, ${ }^{19}$ poly $(3,4$ ethylenedioxythiophene) polystyrene sulfonate, ${ }^{20,21}$ poly(3-hexylthiophene) $)^{22}$ and polyaniline (PANi) ${ }^{10-12}$ PANi is one of most interesting sensing materials due to its ease of synthesis. It is also stable and has tunable electrical property obtained by using different redox process. ${ }^{11,13,23}$ The gas sensing mechanism relies on the presence of $\pi-\pi$ conjugated electrons in conducting polymer chains. ${ }^{\mathbf{1 4}}$ The interaction of gas molecules with PANi will change carrier densities in the polymer. ${ }^{23}$ Its electrical conductivity will change depending on the type of gas molecules being monitored. ${ }^{24}$ There are many studies on the conductivity of PANi on substrates obtained by various techniques such as the polymerization process, ${ }^{\mathbf{1 1}}$ electrospun method, ${ }^{\mathbf{1 2}} \mathrm{dip}$ coating $^{24}$ or printing techniques. ${ }^{25} \mathrm{PANi}$ is one of the polymer which can detect ammonia $\left(\mathrm{NH}_{3}\right)$ gas, one of a toxic gas. The detectable concentration level of $\mathrm{NH}_{3}$ is another aspect that needs to be consider. $\mathrm{NH}_{3}$ is a colorless and pungent odor that humans can detect unaided at concentration greater than
Pathumthani 12121, Thailand. E-mail: jrawat@tu.ac.th

${ }^{b}$ School of Advanced Materials Science and Engineering, Sungkyunkwan University, Suwon 440746, Korea

${ }^{c} S K K U$ Advanced Institute of Nanotechnology (SAINT), Sungkyunkwan University, Suwon 440746, Korea

${ }^{d}$ Department of Materials Science, Faculty of Science, Kasetsart University, Bangkok 10900, Thailand. E-mail: nattasamon.p@ku.ac.th

${ }^{e}$ Specialized Center of Rubber and Polymer Materials for Agriculture and Industry (RPM), Faculty of Science, Kasetsart University, Bangkok 10900, Thailand

$\dagger$ Electronic supplementary information (ESI) available. See DOI: 10.1039/c9ra04005f 
$5 \mathrm{ppm}$. Concentrations of $\mathrm{NH}_{3}$ above $50 \mathrm{ppm}$ can cause health problems. ${ }^{26}$ The recommended exposure limit (REL) for $\mathrm{NH}_{3}$ set by the National Institute for Occupational Safety and Health Administration (NIOSH) is $25 \mathrm{ppm}$ for an 8 hour time-weighted average (TWA), while the short-term exposure limit (STEL) of $35 \mathrm{ppm}$ during any 15 minute period in a work day. ${ }^{27-30}$ There are some studies that reported the used of PANi as sensing material for textile-based $\mathrm{NH}_{3}$ gas sensor. ${ }^{31,32}$ The polymerization method was often used for PANi fabrication on textile ${ }^{31,32}$ or flexible substrate. ${ }^{33}$ The process was done with many parameters controlling. However, to avoid complicated conditions, coating process is a considerable method since it is easy, convenient and has high possibility to be commercialized.

In this research, we constructed a new type of single-yarnbased gas sensor that can be sewable and be reused as a low concentration $\mathrm{NH}_{3}$ detection. The material PANi was used as the conductive and sensitive layers incorporated into a single yarn substrates by a dip coating process. The sensing properties of the sewable yarn sensors were investigated for room temperature operation. We demonstrated that the single yarn gas sensor can act as a variable resistor for adjusting the current through the light emitting diode (LED). This sophisticated applications were accomplished by integrating the gas sensor yarn into a microcontroller system which can communicates what the environment surrounding the clothes. The gas sensor only requires one single yarn in the weave. This makes it convenient for mass production.

\section{Results and discussion}

\section{PANi coating process}

To fabricate the yarn-based $\mathrm{NH}_{3}$ gas sensor, the coating process for PANi as seen in Fig. 1a was first optimized. The concentration of PANi was varied from 1.0, 1.5, 2.0 and $2.5 \mathrm{wt} \%$ as show in Fig. 1b. After doping with hydrochloric acid ( $\mathrm{HCl})$, the electrical resistance of the conductive cotton and rayon yarns decreased significantly with increasing concentrations of PANi solution. At concentrations of 1.0 and $1.5 \mathrm{wt} \%$, the average electrical resistances of cotton and rayon were high with large variation over different parts of the yarn. This indicates that the PANi molecules were not well distributed over the yarns' surface, mainly due to the low concentration of PANi in the coating solution. By increasing the PANi concentration to $2.0 \mathrm{wt} \%$, the average resistance as well as the variation was considerably reduced. Particularly, the PANi-coated cotton and rayon exhibited average resistance values of $19.4 \pm 8.9 \mathrm{k} \Omega \mathrm{cm}^{-1}$ and $45.2 \pm 21.6$ $\mathrm{k} \Omega \mathrm{cm}^{-1}$, respectively (Fig. S1†). This indicates that PANi molecules in this case are well distributed on the yarn surface. Increasing the concentration to $2.5 \mathrm{wt} \%$ only had slight influence on the resistance and variation. Therefore, further experiments were carried out for the yarn with $2.0 \mathrm{wt} \%$ PANi concentration. In the case of polyester yarn, the resistance could not be measured even after the PANi was coated. This was attributed to the surface nature of polyester yarn which had no hydroxyl $(-\mathrm{OH})$ groups. The cotton and rayon yarns, on the other hand, have a considerable number of $-\mathrm{OH}$ groups on their

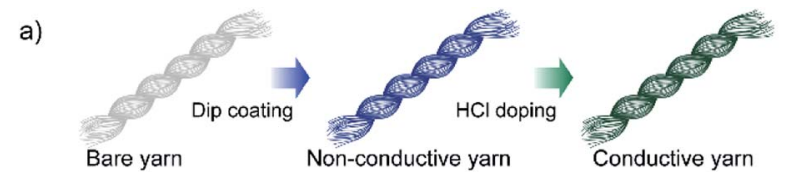

b) c)
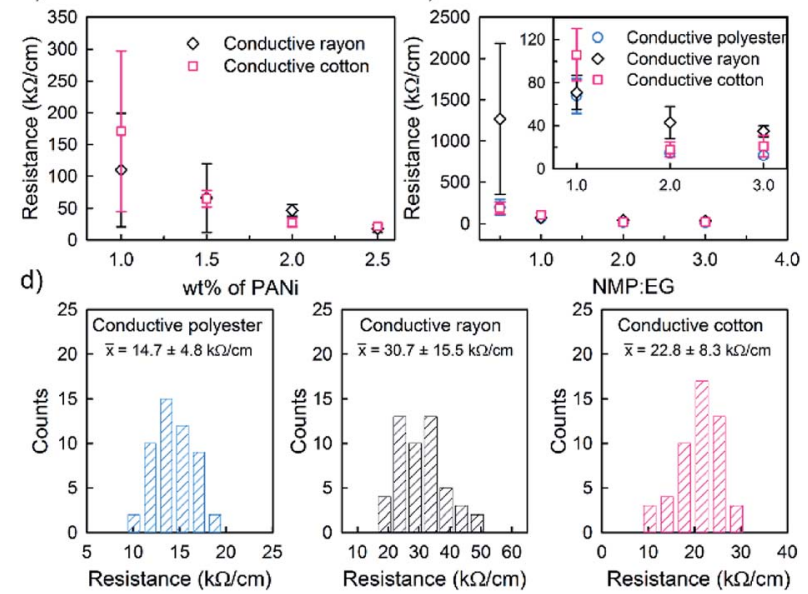

Fig. 1 (a) Fabrication process of the conductive yarn. (b) Electrical resistance investigation by increasing weight percent of PANi. (c) Electrical resistance investigation by increasing ratio of NMP : EG on polyester, cotton and rayon yarns. (d) Histograms of electrical resistance of conductive polyester, cotton and rayon yarns with adding EG.

surfaces, which allow the adsorption of PANi molecules on the surface.

To promote the adhesion of PANi molecules on the polyester yarn surface, we added ethylene glycol (EG) to the coating solution as a adhesion promoter. Since the EG molecule contains the $-\mathrm{OH}$ groups, it can induce the bonding between PANi molecule and the polyester yarn. ${ }^{34}$ To optimize the EG concentration, the ratio of 1-methyl-2-pyrrolidinone to EG (NMP : EG) was varied as $1: 2,1: 1,2: 1$ and $3: 1$ by volume. Fig. 1c shows the electrical resistance of PANi-coated polyester yarns fabricated with different NMP : EG ratios. At first, it can be seen that by adding EG, the conductive polyester yarns could be obtained. This indicated that PANi was successfully coated onto the polyester yarn. Next, as the volume ratio of EG was decreased from $1: 2$ to $1: 1$ and $2: 1$, the electrical resistance decreased from $197.9 \pm 90.7 \mathrm{k} \Omega \mathrm{cm}^{-1}$ to $67.8 \pm 16.4 \mathrm{k} \Omega \mathrm{cm}^{-1}$ and $14.7 \pm 4.8 \mathrm{k} \Omega \mathrm{cm}^{-1}$, respectively. Changing the NMP : EG ratio to $3: 1$ had a negligible effect on the resistance. We therefore set the optimum ratio of NMP : EG to be $2: 1$, in regards to the conductivity. Fig. 1d, left panel shows the electrical resistance distribution of the fabricated conductive polyester yarns (NMP : EG ratio $=2: 1$ ) measured at 50 points. This confirms that the yarns were of reasonable uniformity. In a similar manner, the conductive cotton and rayon yarns were fabricated, by using coating solutions with NMP : EG ratio of $2: 1$. Fig. 1d, middle and right panels show the electrical resistance distributions of conductive cotton and rayon yarns, respectively. Compared to those fabricated without the EG, the resistance values are only slightly different. 
The surface morphologies of PANi-coated conducive yarns are analyzed. Fig. 2 shows the scanning electron microscope (SEM) images of polyester, rayon and cotton yarns with different coating conditions. In the case of rayon and cotton yarns, the bare yarns show smooth surface while the PANi-coated yarns, either with or without using EG, exhibit rough and irregular morphologies. These are indicative of the PANi molecule adsorption on the surface. In the case of polyester yarn, however, no significant change in the surface was observed when the PANi was coated without using EG. By adding EG, on the other hand, uniform and dense layer of PANi was coated on the surface. Additional SEM images can be seen in Fig. S2. $\dagger$

To further identify the existence of PANi layer on yarn surfaces, Fourier-transform infrared spectroscopy (FTIR) with an attenuated total reflection (ATR) was done as shown in Fig. 3. The cotton and rayon show almost the same peaks on both the bare yarns and coated yarns. While the polyester shows different peaks since it has a different structure. The carbonyl $(\mathrm{C}=\mathrm{O})$ stretching band at $1714 \mathrm{~cm}^{-1}$ in the FTIR spectra identify it as polyester. ${ }^{35}$ For PANi molecules, the peaks at $1593 \mathrm{~cm}^{-1}$ and $1498 \mathrm{~cm}^{-1}$ indicate the presence of quinoid and benzenoid ring-stretching, respectively. ${ }^{36,37}$ In ESI Table S1† also shows the functional group relating to its wavenumber $\left(\mathrm{cm}^{-1}\right)$. This clearly reveals and supports the absorption of the PANi molecules on the yarns' surface.

\section{Mechanical properties of PANi-coated conductive yarns}

The influence of the PANi coating on the mechanical properties of conductive yarns are shown in Fig. S3. $\uparrow$ The maximum strain $\left(\varepsilon_{\max }\right)$ of the bare cotton yarn was $15.5 \%$ and is reduced to $13.1 \%$ after coating the PANi layer (before $\mathrm{HCl}$ doping). After $\mathrm{HCl}$ doping, the $\varepsilon_{\max }$ is further reduced to $8.3 \%$. Similar behavior was observed for the rayon yarn. In this case, the bare yarn shows an $\varepsilon_{\max }$ of $15.2 \%$ which is reduced to $14.9 \%$ and $8.4 \%$ after PANi coating and $\mathrm{HCl}$ doping, respectively. The significant reduction of mechanical strength and of the breaking load

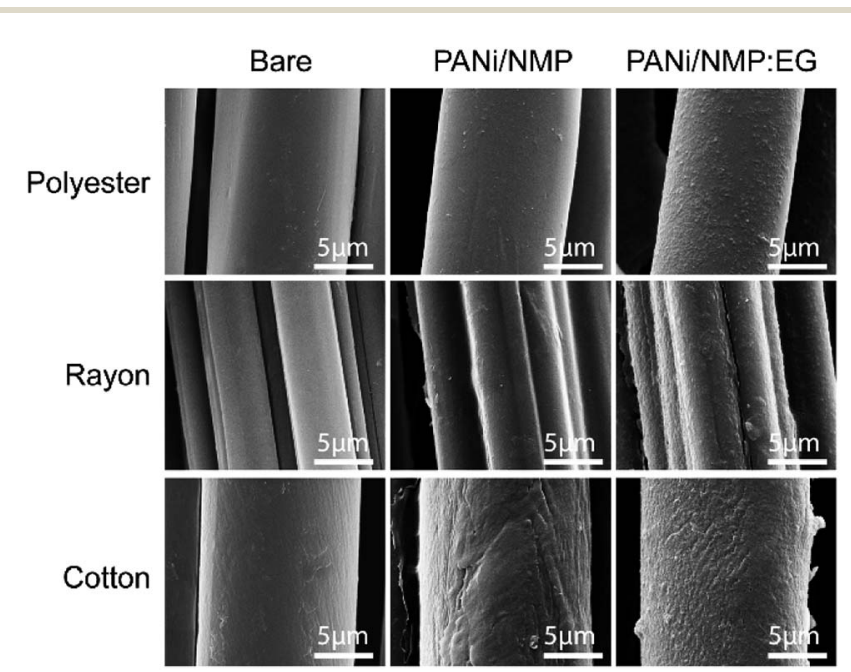

Fig. 2 Comparison of SEM images between bare yarns and coating PANi with/without EG on polyester, rayon and cotton yarns.

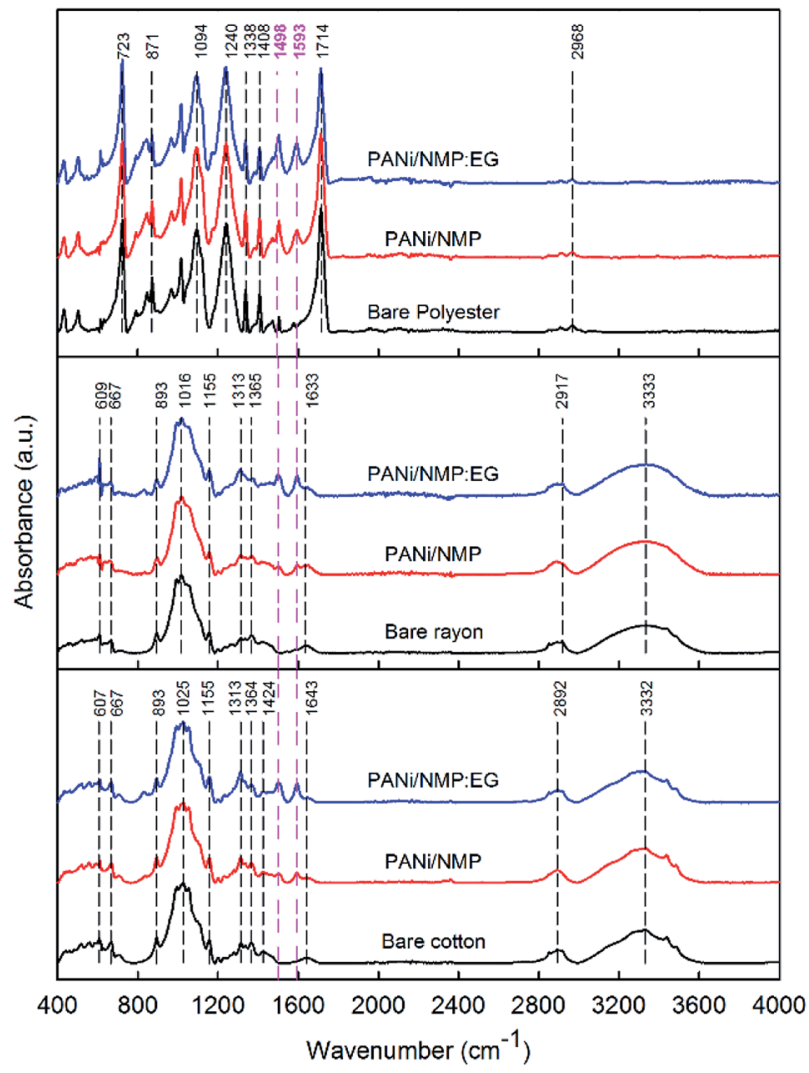

Fig. 3 Comparison FTIR spectra of bare yarns and PANi coating with/ without EG on polyester, rayon and cotton yarns.

observed in cotton and rayon yarns can be attributed to the $\mathrm{HCl}$, which can break the structure of cellulose. ${ }^{38}$ In the case of polyester yarn, however, opposite behavior is observed. Here, the $\varepsilon_{\max }$ was increased from $15.3 \%$ to $19.2 \%$ after the PANi coating. Even after the $\mathrm{HCl}$ doping, the $\varepsilon_{\max }$ is still higher than that of the bare yarn.

\section{Gas sensing properties of PANi-coated conductive yarns}

Using the fabricated conductive yarns, textile-based gas sensors were fabricated as shown in Fig. 4a. The PANi-coated conductive yarn was used as a resistance load connection between the buttons on cloth, one side of the button is attached to a LED and the other side attached to a battery. Linear and almost symmetrical current-voltage $(I-V)$ characteristics of the gas sensor units indicate that ohmic contacts are formed between the conductive yarn and the metal electrodes (Fig. 4b). The sensing response $(S)$ was calculated from the gas response curve by using $S=\left(R_{\mathrm{g}}-R_{0}\right) / R_{0}$, where, $R_{0}$ and $R_{\mathrm{g}}$ are sensing resistance before and after exposure to a target gas. Since the conductive polyester yarn exhibited better mechanical properties than the other conductive yarns, it was first tested to examine the sensitivity to different toxic gases such as ammonia $\left(\mathrm{NH}_{3}\right)$, nitrogen dioxide $\left(\mathrm{NO}_{2}\right)$, carbon monoxide $(\mathrm{CO})$, hydrogen disulfide $\left(\mathrm{H}_{2} \mathrm{~S}\right)$ and sulfur dioxide $\left(\mathrm{SO}_{2}\right)$. Fig. $4 \mathrm{c}$ and $\mathrm{S} 4 \mathrm{a} \uparrow$ show the dynamic sensing response on each toxic gas at concentrations of $50 \mathrm{ppm}$. It appears that the sensor exhibited the highest 
a)
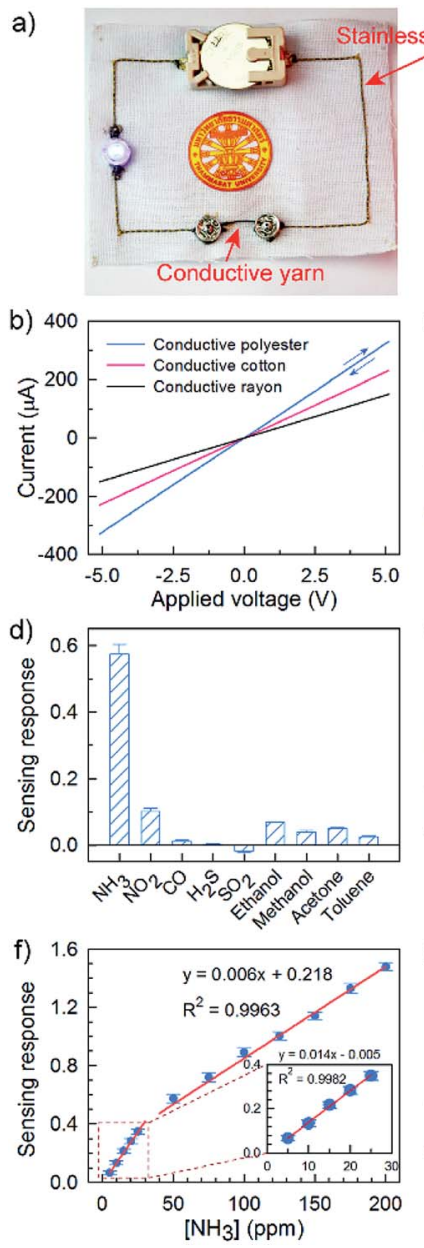

Fig. 4 (a) Testing circuit images of gas sensor yarns. (b) Currentvoltage $(I-V)$ characteristic of textile sensors on different yarn substrate. (c) Sensing response of conductive polyester yarn sensor on different toxic gases exposure at $50 \mathrm{ppm}$. (d) Selectivity to different toxic gases exposure at $50 \mathrm{ppm}$ and different volatile organic compounds exposure at $200 \mathrm{ppm}$. (e) Sensing response comparison of different yarn substrates on $50 \mathrm{ppm} \mathrm{NH}_{3}$ gas. (f) Gas sensing response from $5 \mathrm{ppm}$ to $200 \mathrm{ppm}$ of ammonia gas concentrations on conductive polyester yarn sensor (inset: the linear sensing responses were shown at $5 \mathrm{ppm}$ to $25 \mathrm{ppm}$ ). (g) Stability of sensing response (blue circle) measuring within 60 days of conductive polyester yarn sensor (inset: dynamic response under repeated $50 \mathrm{ppm} \mathrm{NH}_{3}$ gas exposure).

sensitivity to $\mathrm{NH}_{3}$ gas among the toxic gases tested. Its sensitivity was comparable to the volatile organic compounds such as ethanol, methanol, acetone and toluene (at concentrations of $200 \mathrm{ppm}$ ), Fig. S4b. $\dagger$ The sensitivity to $\mathrm{NH}_{3}$ gas was comparably higher (Fig. 4d). These results show that the PANi-coated polyester yarn has a selectivity to $\mathrm{NH}_{3}$ gas and it would be a good candidate for a sensing element for $\mathrm{NH}_{3}$.

In Fig. 4e, the gas response characteristics of three different conductive yarns, polyester, cotton and rayon, are compared, when exposed to $50 \mathrm{ppm}$ of $\mathrm{NH}_{3}$ gas. The conductive polyester yarn exhibited the highest response up to $57 \%$, while the conductive cotton and rayon yarns showed a bit lower response of $50 \%$ and $48 \%$, respectively. The low responses for cotton and rayon can be attributed to the low stability of conductive cotton and rayon yarns under dark and dry air $\left(30 \% \mathrm{RH}, 25{ }^{\circ} \mathrm{C}\right.$, Fig. S5†), leading to a high base-resistance and a low sensing response under gas exposure. In addition, compared to cotton and rayon yarns, the polyester yarn showed much faster recovery. It is expected that the cellulose structure in cotton and rayon yarns can adsorb the $\mathrm{NH}_{3}$ molecules more closely, ${ }^{39}$ thereby slightly delaying the recovery process after the $\mathrm{NH}_{3}$ gas exposure. The effect of humidity to $\mathrm{NH}_{3}$ gas sensing response was also tested and shown in Fig. S6. $\dagger$ The results indicate that the humidity has a negligible effect on the gas sensing properties.

To investigate the concentration-dependent sensing properties of polyester yarn sensors, the sensing response at different $\mathrm{NH}_{3}$ gas concentrations was measured in the range of 5-200 ppm (Fig. 4f). In the range of 5-25 ppm, the polyester yarns sensor exhibited a good linearity against the $\mathrm{NH}_{3}$ concentration $\left(R^{2}=0.9982\right)$ and gas sensitivity of $0.014 \mathrm{ppm}^{-1}$. From the extrapolation of the line, the minimum detection limit was $0.4 \mathrm{ppm}$. Above $50 \mathrm{ppm}$, the sensitivity was slightly reduced $\left(0.006 \mathrm{ppm}^{-1}\right)$ which can be attributed to a high surface coverage of $\mathrm{NH}_{3}$ molecules attached on the PANi. Considering the human's detection level for $\mathrm{NH}_{3}$ gas is above $5 \mathrm{ppm}$ and the recommended exposure limit ( $25 \mathrm{ppm}),{ }^{26,28}$ the polyester yarn sensor would be a good candidate for real-time monitoring of $\mathrm{NH}_{3}$ gas.

For the practical application of polyester yarn-based gas sensors, the long-term operational stability was also examined over a period of 60 days. As shown in Fig. 4g, the sensor responses were measured for every 5 days. The sensing response showed relatively stable characteristics and only a slight decrease in the sensing response was observed $(8.6 \%$ after 60 days). In addition, a repeated dynamic response of polyester yarn sensor was also investigated by exposing the $\mathrm{NH}_{3}$ gas (50 ppm) at an interval of $30 \mathrm{~min}$. The $\mathrm{NH}_{3}$ gas was tuned on for $10 \mathrm{~min}$. As shown in the inset of Fig. $4 \mathrm{~g}$, the sensor showed reasonably good and reproducible response behavior to the repeated $\mathrm{NH}_{3}$ gas exposure.

Concerning the $\mathrm{NH}_{3}$ gas sensing mechanism using PANi, a schematic illustration is shown in Fig. S7.† The sensing mechanism can describe based on a change of polaron density inside the band gap of polymer. ${ }^{13}$ In particular, polyaniline emeraldine base (PANi-EB) contains nitrogen $(\mathrm{N})$ atoms attach to quinoid rings, and these $\mathrm{N}$ atoms can interact with $\mathrm{HCl}$ which is an oxidizing agent. Once the nitrogen atoms get protonated from $\mathrm{HCl}$, as show in eqn (1), yielding conducive form known as the polyaniline emeraldine salt (PANi-ES). The free positive charge was created and can move along the polymer backbone. The coupling between mobile carriers and polarons on the PANi backbone are responsible for conductivity in PANi-ES. ${ }^{13}$ PANi-ES is a p-type semiconductor and under the conduction state, after the appearance of PANi- $\mathrm{H}^{+}$in the backbone, it can detect $\mathrm{NH}_{3}$ gas which is well-known as a reducing gas. Upon expose to $\mathrm{NH}_{3}$ gas, electrons are transferred from $\mathrm{NH}_{3}$ to $\mathrm{PANi}-\mathrm{H}^{+}$, which then reduce hole-carrier concentration in the PANi-ES resulting in higher in resistance of the backbone. In addition, this creates lower amount of polarons and thus increase the electrical resistance. ${ }^{25}$ Also, after the $\mathrm{NH}_{3}$ molecule losses the electron, it 
behaves as $\mathrm{NH}_{4}{ }^{+}$ion attach to the backbone. The electron donation and reversible process of PANi-ES and $\mathrm{NH}_{3}$ gas can occur due to the redox reaction ${ }^{23}$ as seen in eqn (2). When the air flows to the system, the amount of the $\mathrm{NH}_{3}$ is gradually diluted yielding a lower amount of donated electrons to PANi- $\mathrm{H}^{+}$. This makes the hole carrier concentration also gradually increased back to what it was before and resulting in the original value of PANi-ES conductivity. This corresponds to results shown in Fig. $4 \mathrm{e}$ and the inset of Fig. $4 \mathrm{~g}$.

$$
\begin{gathered}
\mathrm{PANi}+\mathrm{HCl} \rightarrow \mathrm{PANi}-\mathrm{H}^{+}+\mathrm{Cl}^{-} \\
\mathrm{PANi}^{-} \mathrm{H}^{+}+\mathrm{NH}_{3} \rightleftharpoons \mathrm{PANi}+\mathrm{NH}_{4}^{+}
\end{gathered}
$$

We also investigated the effect of bending angle on the gas sensing properties. Here, the bending angle was changed from $0^{\circ}$ to $45^{\circ}, 90^{\circ}$ and $135^{\circ}$, and the sensing responses were observed. As shown in Fig. S8, $\dagger$ no significant variation was observed even at an angle of $135^{\circ}$, suggesting that the fabricated gas sensors are mechanically flexible and stable.

\section{Real-time monitoring of $\mathrm{NH}_{3}$ gas using polyester yarn sensors}

In order to demonstrate a possible application for textile-based gas sensor, an $\mathrm{NH}_{3}$ gas sensing unit was fabricated on a fabric using the PANi-coated polyester yarn. As shown in Fig. 5a, the polyester yarn sensor was sewn on the fabric in a triangular pattern (a total length of $1.5 \mathrm{~cm}$ ). Stainless steel threads were used as electrical contacts on both ends of the yarn. For the gas sensing unit, three-trace level of $\mathrm{NH}_{3}$ gas was designed using a simple comparator circuit, which indicates the exposure level by LEDs. As shown in Fig. 5b, under clean atmosphere, no LEDs

a)

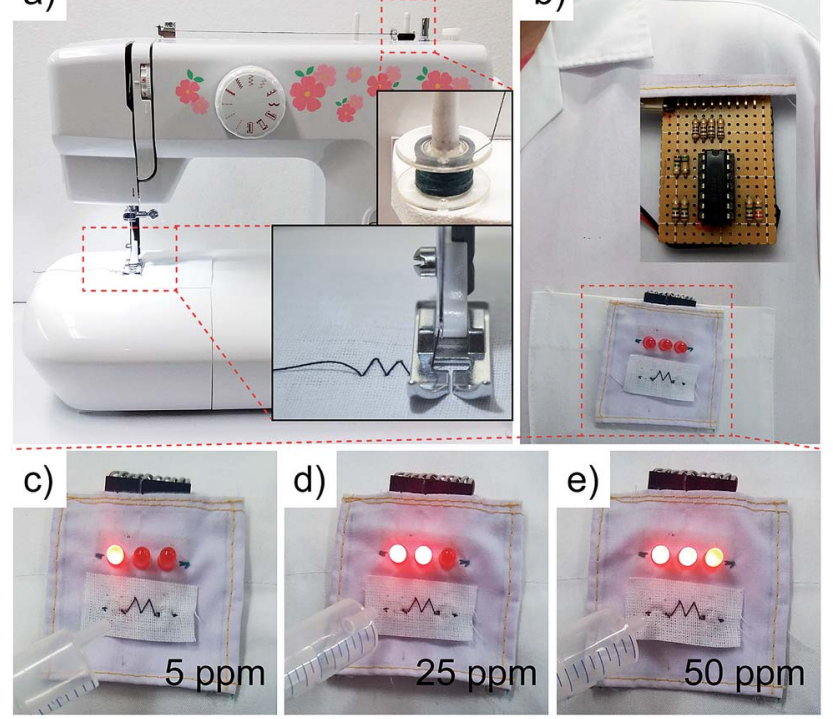

Fig. 5 Photograph images of conductive polyester yarn sensor. (a) Sewing conductive polyester yarn on cloth by using a sewing machine. (b) Polyester yarn sensor attached on a pocket of laboratory coat. (c-e) Gas sensor demonstration with different concentration of ammonia gas at 5 ppm, 25 ppm and 50 ppm by turning on LEDs. were turned on. However, when the $\mathrm{NH}_{3}$ gas concentration becomes higher than 5 ppm, one LED is turned on (Fig. 5c). When the $\mathrm{NH}_{3}$ gas concentration is higher than $25 \mathrm{ppm}$ and $50 \mathrm{ppm}$, two and three LEDs turn on as seen in Fig. 5d and e, respectively. These results clearly show that the fabricated PANicoated polyester yarn can be utilized in real-time monitoring of $\mathrm{NH}_{3}$ gas, indicating the exposure levels to the users. By integrating the polyester yarn sensor with microcontroller systems, an active gas sensor for wireless applications can be realized. In Fig. S9a, $\dagger$ we showed a wireless wearable $\mathrm{NH}_{3}$ gas monitoring system, in which the polyester yarn sensor was sewn on the upper arm of a shirt. The sensor was connected to a microcontroller system integrated with a wireless transmitter and receiver circuit. As illustrated in Fig. $\mathrm{S} 9 \mathrm{~b}, \dagger$ when the $\mathrm{NH}_{3}$ gas in the environment reaches a limit, the system sends an alarm signal to the user. The wireless gas sensing system can also be used to monitor the gas level continuously over a given period of time. Here, when the sensor is exposed to a certain level of $\mathrm{NH}_{3}$ gas, the resistance of the sensor changes which results in a voltage drop (Fig. S9c $\dagger$ ). As the exposed gas level is increased, the voltage drop will become greater.

The performance of our yarn sensor is compared with other works in Table 1. It can be noted that the resistance value of a yarn sensor is an important factor to determine the cost effectiveness of the sensor circuit. Due to low based resistance is necessary for a simple amplifier circuit and low level input impedance of measurement devices. Our polyester sensor had a resistance value of $0.015 \mathrm{M} \Omega \mathrm{cm}^{-1}$ which is the lowest compare to other PANi-based yarn sensors and it is only 2 times larger than that of SWCNT-oncotton which had a value of $0.008 \mathrm{M} \Omega \mathrm{cm}^{-1}$. There are also a few reports show stability testing on yarn sensors because it is another importance factor to identify durability in real use. Our yarn sensor showed a stability up to 60 days which is relatively good compared to other PANi-based $\mathrm{NH}_{3}$ sensors. Our intension to fabricate low cost sewable gas sensor for commercialize make us conduct sensor response after pulling our yarn sensor with a commercial sewing machine. The pulling force from the sewing machine has no effect to sensing response since the results of our yarn sensor confirm its higher value of strain/force after treatment with chemicals. The other importance parameter is area detection, this is essential for making array gas detector. Our sensor can perform under small area compare to the other yarn sensors.

\section{Experimental section}

\section{Materials}

Polyaniline emeraldine base (PANi-EB, $M_{\mathrm{w}} \sim 20000$ ), 1-methyl2-pyrrolidinone 99\% (NMP) and ethylene glycol (EG) 99.8\% were purchased from Sigma Aldrich. Hydrochloric acid 37\% ( $\mathrm{HCl})$, acetone and all solvents were purchased from RCI Labscan. All toxic gases, inert gases and synthetic air were purchased from Linde. All chemicals were used as received.

\section{Fabrication of conductive yarns}

The conductive yarns were fabricated on single yarn substrates in ambient environment by dip coating process. In brief, the 
Table 1 Comparison of the performance of the yarn sensors ${ }^{a}$

\begin{tabular}{|c|c|c|c|c|c|c|c|}
\hline Materials & Substrates & Gas & $R_{0}(\mathrm{M} \Omega)$ & Strain $(\%) /$ force $(\mathrm{N})$ & Stability (days) & Detection area $\left(\mathrm{mm}^{2}\right)$ & Ref. \\
\hline PANi & Yarn/PS & $\mathrm{NH}_{3}$ & $0.015 / \mathrm{cm}$ & $17 / 10$ & 60 & 9.42 & This work \\
\hline PANi & Yarn/PAN & $\mathrm{NH}_{3}$ & $0.2 / \mathrm{cm}$ & $5 / 0.51$ & 30 & 5.65 & 31 \\
\hline PANi/MWCNT & Fabric/- & $\mathrm{NH}_{3}$ & $2.28 / \mathrm{cm}^{2}$ & - & 30 & 100 & 32 \\
\hline SWCNT & Yarn/CT & $\mathrm{NH}_{3}$ & $0.008 / \mathrm{cm}$ & $14 /-$ & - & - & 9 \\
\hline PPy & Fabric/CT & $\mathrm{NH}_{3}$ & - & - & - & - & 41 \\
\hline
\end{tabular}

${ }^{a}$ MWCNT is multiwall carbon nanotube; SWCNT is single wall carbon nanotube; PPy is polypyrrole; RGO is reduced graphene oxide; PAN is polyacrylonitrile; NW-PP is non-woven polypropylene.

precursor solution of conductive and sensitive layers was prepared by dissolving PANi-EB $(32.3 \mathrm{mg}, \sim 1.6 \mu \mathrm{mol})$ in 1 methyl-2-pyrrolidinone $(1 \mathrm{~mL}, 10 \mathrm{mmol})$ yielding a concentration of $2 \mathrm{wt} \%$. The PANi-EB/NMP solution was stirred for 72 hours at room temperature. To enhance the adhesion of PANi on the yarn surfaces, ethylene glycol $(0.5 \mathrm{~mL}, 8.97 \mathrm{mmol})$ was slowly dropped into the PANi-EB/NMP solution using a mixing ratio of $2: 1$ (NMP : EG). A single polyester yarn substrate was sonicated using acetone and dried in oven at $60{ }^{\circ} \mathrm{C}$ for 30 minutes to remove any surface residues. After that, the cleaned yarn was immersed into the PANi-EB/NMP : EG solution for 10 minutes and dried at $60^{\circ} \mathrm{C}$ for 2 hours. Finally, the PANi-coated yarn was doped with $5 \mathrm{M} \mathrm{HCl}$ for 30 minutes to induce a conductive form of polyaniline emeraldine salt (PANi-ES). Identical process was also carried out for rayon and cotton yarns (Fig. 1a).

\section{Fabrication of textile-based gas sensors and circuits}

For application of all textile-based gas sensor, the PANi-coated yarn was sewn onto the fabric by using a sewing machine. Conducting stainless-steel threads were used as the electrode connector for measurement purposes. For fabrication of real-time gas monitoring devices, the sensing signal was wired to an input signal of three-level comparator circuits (LM324 operation amplifier) which was connected to a small rechargeable battery $3.7 \mathrm{~V}$. LED was used as the alarm devices. For wireless gas monitoring, a microcontroller (NodeMCU Devkit V1.0) was used instead.

\section{Characterization of gas sensors}

The morphologies of yarn-based gas sensors were observed by using a scanning electron microscope (SEM, FEI model Quanta 450). The deposition of PANi on yarn surfaces was verified by Fourier-transform infrared spectroscopy (FTIR, Bruker model Vertex 70) with an attenuated total reflection (ATR) setup. The electrical characteristics of the sensors were measured by Keithley 6517B electrometer and Keithley 2400 source meter. The gas sensing characteristics were analyzed using a homebuilt gas measurement system. Briefly, the conductive yarn sensor was placed in a closed chamber and a constant voltage of $5 \mathrm{~V}$ was supplied to the sensor. Desired concentration of $\mathrm{NH}_{3}$ gas was obtained by diluting $500 \mathrm{ppm}$ standard gas with synthetic air before feeding into the measurement chamber. The total gas flow rate was set at $150 \mathrm{sccm}$ by programmable mass flow controller. In addition, the operating temperature and humidity inside the chamber were measured using a commercial sensor (SHT15, Sensirion AG). The tensile strength of the yarns was investigated using an automatic tensile tester. Here, the load cell was $50 \mathrm{~N}$ and the sample length was $10 \mathrm{~cm}$ with a pulling speed of $10 \mathrm{~mm} \mathrm{~min}$.

\section{Conclusions}

In summary, a single-yarn-based gas sensor with a highly mechanical and sewable textile sensor as well as clothesbased sensing system has been shown for the first time. A chemiresistive type of textile-based gas sensor was fabricated by coating PANi on the surface of common yarns such as cotton, rayon and polyester. EG was used as a mediator to help PANi attached to the yarns surface. This is possible due to the existence of -OH group in the EG. By doping PANi with $\mathrm{HCl}$, the conductive yarns were achieved. The results show that the conductive polyester yarn exhibits a higher sensing response with a good mechanical strength when compared to those of conductive cotton and conductive rayon yarns. The conductive polyester yarn also exhibits the highest response to $\mathrm{NH}_{3}$ gas compared the response to other toxic gases. $\mathrm{NH}_{3}$ gas can be measured to as low as $5 \mathrm{ppm}$ and up to more than $200 \mathrm{ppm}$ under using the same conductive yarn. This means that the gas can be sensed repeatedly and this textile-based gas sensor is reusable. More advantage of this sensor, its light weight and its sewable into a fabric using a sewing machine. The results show that the conductive polyester yarn-based gas sensor has the advantage of being able to be incorporate into smart gas monitoring shirts use.

\section{Conflicts of interest}

There are no conflicts to declare.

\section{Acknowledgements}

This work was partially supported by Thammasat University through the National Research Center of Thailand (Contract No. 
$17 / 2559$ and 16/2560), Thammasat University innovation scholar (Contract No. 2/2559) and Faculty of Science and Technology (Contract No. 11/2559). N. P. thanks Kasetsart University Research and Development Institute (KURDI) and the Faculty of Science, Kasetsart University. We thank the Scientific Equipment Center and Department of Materials Science at Faculty of Science, Kasetsart University for using their SEM and FTIR equipment.

\section{Notes and references}

1 D. J. Lipomi, B. C. K. Tee, M. Vosgueritchian and Z. Bao, Adv. Mater., 2011, 23, 1771.

2 Y. M. Choi, M. G. Lee and Y. Jeon, Energies, 2017, 10, 1.

3 C. Li, M. Islam, J. Moore, J. Sleppy, C. Morrison, K. Konstantinov, S. X. Dou, C. Renduchintala and J. Thomas, Nat. Commun., 2016, 7, 1.

4 Z. Chai, N. Zhang, P. Sun, Y. Huang, C. Zhao, H. J. Fan, X. Fan and W. Mai, ACS Nano, 2016, 10, 9201.

5 A. E. Ostfeld, A. M. Gaikwad, Y. Khan and A. C. Arias, Sci. Rep., 2016, 6, 1.

6 J. C. Y. Kenry and C. T. Lim, Microsyst. Nanoeng., 2016, 2, 1.

7 Y. J. Yun, W. G. Hong, D. Y. Kim, H. J. Kim, Y. Jun and H. K. Lee, Sens. Actuators, B, 2017, 248, 829.

8 Y. J. Yun, W. G. Hong, N. J. Choi, B. H. Kim, Y. Jun and H. K. Lee, Sci. Rep., 2015, 5, 1.

9 J. W. Han, B. Kim, J. Li and M. Meyyappan, Appl. Phys. Lett., 2013, 102, 1.

10 Z. Ma, P. Chen, W. Cheng, K. Yan, L. Pan, Y. Shi and G. Yu, Nano Lett., 2018, 18, 4570.

11 S. Chen and G. Sun, ACS Appl. Mater. Interfaces, 2013, 5, 6473.

12 Y. Zhang, J. J. Kim, D. Chen, H. L. Tuller and G. C. Rutledge, Adv. Funct. Mater., 2014, 24, 4005.

13 S. Virji, J. Huang, R. B. Kaner and B. H. Weiller, Nano Lett., 2004, 4, 491.

14 H. Bai and G. Shi, Sensors, 2007, 7, 267.

15 J. Janata and M. Josowicz, Nat. Mater., 2003, 2, 19.

16 M. E. Abdelhamid, A. P. O'Mullane and G. A. Snook, RSC $A d v .$, 2015, 5, 11611.

17 J. G. Ibanez, M. E. Rincón, S. Gutierrez-Granados, M. Chahma, O. A. Jaramillo-Quintero and B. A. FrontanaUribe, Chem. Rev., 2018, 118, 4731.

18 M. Naseri, L. Fotouhi and A. Ehsani, Chem. Rec., 2018, 18, 599.

19 J. B. Chang, V. Liu, V. Subramanian, K. Sivula, C. Luscombe, A. Murphy, J. Liu and J. M. J. Fréchet, J. Appl. Phys., 2006, 100, 1.

20 M. Hakimi, A. Salehi and F. A. Boroumand, IEEE Sens. J., 2016, 16, 6149.
21 J. Eom, R. Jaisutti, H. Lee, W. Lee, J. Heo, J. Lee, S. K. Park and Y. Kim, ACS Appl. Mater. Interfaces, 2017, 9, 10190.

22 M. R. Cavallari, J. E. E. Izquierdo, G. S. Braga, E. A. T. Dirani, M. A. Pereira-da-Silva, E. F. G. Rodríguez and F. J. Fonseca, Sensors, 2015, 15, 9592.

23 H. Ullah, A. A. Shah, S. Bilal and K. Ayub, J. Phys. Chem. C, 2013, 117, 23701.

24 D. Nicolas-debarnot and F. Poncin-epaillard, Anal. Chim. Acta, 2003, 475, 1.

25 K. Crowley, A. Morrin, A. Hernandez, E. O. Malley, P. G. Whitten, G. G. Wallace, M. R. Smyth and A. J. Killard, Talanta, 2008, 77, 710.

26 NIOSH (National Institute for Occupational Safety and Health), Occupational safety and health guidelines for chemical hazards. Supplement III-OHG Revised Guideline for Ammonia, 1992.

27 M. J. Fedoruk and B. D. Kerger, J. Exposure Anal. Environ. Epidemiol., 2005, 15, 534.

28 New Jersey Department of Health, Hazardous substance fact sheet: Ammonia (RTK substance No. 0084) Revision, Right to Know, 2016.

29 U.S. Department of Health and Human Services, Public Health Registry and Agency for Toxic Substances and Disease Registry, Toxicological Profile for Ammonia, 2004.

30 Integrated Risk Information System, National Center for Environmental Assessment, Office of Research and Development and U.S. Environmental Protection Agency, Toxicological Review of Ammonia Noncancer. Inhalation: Executive Summary [CASRN 7664-41-7], Washington, 2016.

31 S. Wu, P. Liu, Y. Zhang, H. Zhang and X. Qin, Sens. Actuators, B, 2017, 252, 697.

32 D. Maity and R. T. R. Kumar, ACS Sens., 2018, 3, 1822.

33 J. Cai, C. Zhang, A. Khan, C. Liang and W. Li, RSC Adv., 2018, 8,5312 .

34 W. Wardencki and A. H. H. Tameesh, J. Chem. Technol. Biotechnol., 1981, 31, 86.

35 P. Peets, I. Leito, J. Pelt and S. Vahur, Spectrochim. Acta, Part A, 2017, 173, 175.

36 E. N. Konyushenko, S. Reynaud, V. Pellerin, M. Trchová, J. Stejskal and I. Sapurina, Polymer, 2011, 52, 1900.

37 M. Trchová, I. Šeděnková, E. Tobolková and J. Stejskal, Polym. Degrad. Stab., 2004, 86, 179.

38 J. H. Lin, Y. H. Chang and Y. H. Hsu, Food Hydrocolloids, 2009, 23, 1548.

39 M. Wada, Y. Nishiyama and P. Langan, Macromolecules, 2006, 39, 2947.

40 J. Qi, X. Xinxin, X. Liu and K. T. Lau, Sens. Actuators, B, 2014, 202, 732.

41 S. C. Deogaonkar and N. V. Bhat, Fibers Polym., 2015, 16, 1803. 